\title{
On additive bases
}

\author{
by \\ W. GaO (Beijing) and Y. O. Hamidoune (Paris)
}

1. Introduction. By $p$ we shall denote a prime number. The group of integers modulo $n$ will be denoted by $\mathbb{Z}_{n}$. Let $G$ be an abelian group and let $S$ be a subset of $G$. As usual, we write

$$
\Sigma(S)=\left\{\sum_{x \in A} x \mid A \subset S\right\} .
$$

The critical number of $G$, denoted by $c(G)$, is the smallest $s$ such that $\Sigma(S)=G$ for every subset $S$ of $G$ with cardinality $s$ not containing 0 .

The parameter $c(G)$ was first studied by Erdös and Heilbronn in [4]. They obtained the inequality $c\left(\mathbb{Z}_{p}\right) \leq 3 \sqrt{6 p}$. Olson proved in $[13]$ that $c\left(\mathbb{Z}_{p}\right) \leq$ $\sqrt{4 p-3}+1$. The authors of [1] obtained the inequality $c\left(\mathbb{Z}_{p}\right) \leq \sqrt{4 p-7}$.

The evaluation of $c(G)$ for groups with composite order was first considered by Mann and Olson. They obtained the inequality $c\left(\mathbb{Z}_{p} \oplus \mathbb{Z}_{p}\right) \leq 2 p-1$ in [11]. Mann and Wou proved that $c\left(\mathbb{Z}_{p} \oplus \mathbb{Z}_{p}\right)=2 p-2$ in [12]. Diderrich proved in [2] the inequality $p+q-2 \leq c(G) \leq p+q-1$, where $G$ is an abelian group of order $p q$ and $q$ is a prime. He conjectured that $c(G)=|G| / p+p-2$ if $|G| / p$ is composite, where $p$ is the smallest prime dividing $|G|$. This conjecture is proved by Diderrich and Mann in [3] for $p=2$. Peng [15] proved Diderrich's conjecture if $G$ is the additive group of a finite field. Lipkin [9] obtained a proof of this conjecture in the case of cyclic groups with large order. This conjecture is proved by one of the present authors in [5] for $p \geq 43$ and by the authors of [8] for $p=3$.

In this paper we achieve the evaluation of $c(G)$, solving the above mentioned conjecture.

2. Some tools. Recall the following well known and easy lemma.

Lemma 2.1 [10]. Let $G$ be a finite group. Let $X$ and $Y$ be subsets of $G$ such that $X+Y \neq G$. Then $|X|+|Y| \leq|G|$.

1991 Mathematics Subject Classification: 11B75, 20 K09. 
We use the following result.

Lemma 2.2 [2]. Let $p, q$ be two primes and let $G$ be an abelian group with order pq. Let $S$ be a subset of $G$ such that $0 \notin S$ and $|S|=p+q-1$. Then $\Sigma(S)=G$.

Let $G$ be an abelian group. Let $B \subset G$ and $x \in G$. As usual, we write $\lambda_{B}(x)=|(B+x) \backslash B|$. For any $B, x$, Olson proved in $[13,14]$

$$
\lambda_{B}(x)=\lambda_{B}(-x)
$$

and

$$
\lambda_{B}(x)=\lambda_{G \backslash B}(x) .
$$

We use the following property which is implicit in [13]: Let $G$ be a finite abelian group. Let $S$ be a subset of $G$ such that $0 \notin S$. Put $B=\Sigma(S)$. For every $y \in S$, we have

$$
|\Sigma(S)| \geq|\Sigma(S \backslash y)|+\lambda_{B}(y) .
$$

We also use the following result of Olson.

Lemma 2.3 (Olson [14]). Let $G$ be an abelian group and let $S$ be a generating subset of $G$ such that $0 \notin S$. Let $B$ be a subset of $G$ such that $|B| \leq|G| / 2$. Then there is $x \in S$ such that

$$
\lambda_{B}(x) \geq \min ((|B|+1) / 2,(|S \cup-S|+2) / 4) .
$$

This result follows, using (1), by applying Lemma 3.1 of [14] to $S \cup-S$. in $[6]$.

We use the following lemma which is a consequence of the main result

LEMma 2.4 [6]. Let $S$ be a subset of an abelian group $G$ such that $S \cap-S$ $=\emptyset$. Then

$$
|\Sigma(S)| \geq 2|S| .
$$

The proof follows easily by induction. Set $B=\Sigma(S)$. By Lemma 2.3 applied to $B$ or $G \backslash B$ and using (2), there is $s \in S$ such that $\lambda_{B}(s) \geq 2$. By $(3),|B| \geq|\Sigma(S \backslash x)|+2 \geq 2|S|$.

3. The main result. Let $X$ be a subset of $G$ with cardinality $k$. Let $\left\{x_{i} ; 1 \leq i \leq k\right\}$ be an ordering of $X$. For $0 \leq i \leq k$, set $X_{i}=\left\{x_{j} \mid 1 \leq\right.$ $j \leq i\}$ and $B_{i}=\Sigma\left(X_{i}\right)$. The ordering $\left\{x_{1}, \ldots, x_{k}\right\}$ will be called a resolving sequence of $X$ if for all $i, \lambda_{B_{i}}\left(x_{i}\right)=\max \left\{\lambda_{B_{i}}\left(x_{j}\right) ; 1 \leq j \leq i\right\}$. The critical index of the resolving sequence is the smallest integer $t$ such that $X_{t-1}$ generates a proper subgroup of $G$.

Clearly, every nonempty subset $S$ not containing 0 admits a resolving sequence. Moreover, the critical index is $\geq 1$. 
We shall write $\lambda_{i}=\lambda_{B_{i}}\left(x_{i}\right)$. By induction we have, using (3), for all $1 \leq j \leq k$,

$$
|\Sigma(X)| \geq \lambda_{k}+\ldots+\lambda_{j}+\left|B_{j-1}\right| .
$$

Put $\delta(m)=0$ if $m$ is odd and $=1$ otherwise. By Lemma 2.3, $\lambda_{i} \geq(i+1+$ $\delta(i)) / 2$ for all $i \geq t$. In particular, for all $s \geq t$,

$$
|\Sigma(X)| \geq(k+s+3)(k-s+1) / 4-1 / 2+\left|B_{s-1}\right| .
$$

THEOREM 3.1. Let $G$ be a finite abelian group with odd order and let $p$ be the smallest prime dividing $|G|$. Let $S$ be a subset of $G$ such that $0 \notin S$ and $|S|=|G| / p+p-2$. If $|G| / p$ is composite, then $\Sigma(S)=G$.

Proof. Set $|G|=n$. One may check easily the result for $n=27$. Suppose $n>27$. Set $k(n)=(n / p+p-2) / 2$. We shall write sometimes $k$ instead of $k(n)$. Clearly we may partition $S=X \cup Y$ so that $|X|=|Y|=k$, $X \cap-X=Y \cap-Y=\emptyset$ and $|\Sigma(X)| \leq|\Sigma(Y)|$.

The result holds by Lemma 2.1 if $|\Sigma(X)|>n / 2$. Suppose the contrary. Since $n$ is odd, we have

$$
|\Sigma(X)| \leq(n-1) / 2 .
$$

Let $\left\{x_{i} ; 1 \leq i \leq k\right\}$ be a resolving sequence for $X$ with critical index $t$.

We first prove that

$$
t \geq 4 \text {. }
$$

Suppose on the contrary that $t \leq 3$. By (5) and (4) applied with $s=3$,

$$
4+(k-2)(k+6) / 4-n / 2 \leq 0 .
$$

Put $f(n)=4+(k(n)-2)(k(n)+6) / 4-n / 2$. Observe that $f^{\prime}(n) \geq 0$. Hence $f(n)$ is increasing as a function of $n$. Since $n \geq p^{3}$, we have by $(7), f\left(p^{3}\right) \leq 0$. Hence $p^{4}-6 p^{3}+5 p^{2}+4 p+4 \leq 0$. It follows that $p=3$. But in this case $n>27$ and hence $n \geq p^{3}+2 p^{2}=45$. It follows that $f(n) \geq f(45)=5 / 2$, contradicting (7).

By Lemma 2.4, $\left|B_{t-1}\right| \geq 2(t-1)$. Obviously $\left|B_{t}\right|=\left|B_{t-1}\right|+\left|x_{t}+B_{t-1}\right|=$ $2\left|B_{t-1}\right| \geq 4(t-1)$.

By (5) and (4), applied with $s=t+1$,

$$
4 t-4+(k-t)(k+t+4) / 4-n / 2 \leq 0 .
$$

Set $F(t, n)=4 t-4+(k(n)-t)(k(n)+t+4) / 4-n / 2$. Notice that $\frac{\partial}{\partial t} F(t, n)=3-t / 2$. Let us show that

$$
t \geq 6 .
$$

Suppose on the contrary that $4 \leq t \leq 5$. Clearly $F(5, n)>F(4, n)$, and $F(4, n)$ is an increasing function of $n$. Now by (8), we have $F\left(4, p^{3}\right) \leq 0$. It follows that $p^{4}-6 p^{3}+5 p^{2}+4 p+52 \leq 0$, a contradiction. 
Let us show that

$$
t \geq n / p^{2}+p-1 \text {. }
$$

Assume the contrary and set $G(n)=F\left(n / p^{2}+p-2, n\right)$. Since $n / p^{2}+p-2 \geq 6$ (we recall that $n>27$ ), we have by $(8)$,

$$
G(n) \leq 0 .
$$

Observe that $G^{\prime}(n)=4 / p^{2}+n /\left(8 p^{2}\right)-1 /(4 p)-n /\left(2 p^{4}\right)-3 / 8 \geq 0$. In particular $G(n)$ is an increasing function. By (11), we have $p^{4}-6 p^{3}-11 p^{2}+$ $132 p-188 \leq 0$, contradicting (11).

Let $H$ be the proper subgroup generated by $X_{t-1}$. Let $p^{\prime}$ be the smallest prime divisor of $n / p$. By (10), $|H \cap S| \geq n /\left(p p^{\prime}\right)+p^{\prime}-1$. If $n / p$ is the product of two primes, then by Lemma 2.2, $\Sigma(S \cap H)=H$. If $n / p$ is the product of more than two primes, then by the induction hypothesis, $\Sigma(S \cap H)=H$.

Since $|H|>n /\left(p p^{\prime}\right)$, we see easily that $q=|G| /|H|$ is a prime. Clearly $|S \backslash H| \geq q-1$. Let $a_{1}, \ldots, a_{q-1}$ be distinct elements from $S \backslash H$. We denote by $\bar{a}_{i}$ the image of $a_{i}$ in $G / H$ under the canonical morphism.

By the Cauchy-Davenport Theorem (cf. [10]), $\left\{0, \bar{a}_{1}\right\}+\ldots+\left\{0, \bar{a}_{p-1}\right\}=$ $G / H$. It follows that $\Sigma\left(a_{1}, \ldots, a_{p-1}\right)+H=G$. The theorem now follows since $\Sigma(S \cap H)=H$.

Acknowledgements. This joint paper was written when the first author held an Austrian Lise Meitner Fellowship (Project No. M00397-MAT). He would like to thank the FWF for the assistance and Alfred Geroldinger for his hospitality.

\section{References}

[1] J. A. Dias da Silva and Y. O. Hamidoune, Cyclic subspaces of Grassmann derivations, Bull. London Math. Soc. 26 (1994), 140-146.

[2] G. T. Diderrich, An addition theorem for abelian groups of order $p q$, J. Number Theory 7 (1975), 33-48.

[3] G. T. Diderrich and H. B. Mann, Combinatorial problems in finite abelian groups, in: A Survey of Combinatorial Theory, J. L. Srivasta et al. (eds.), North-Holland, Amsterdam, 1973, 95-100.

[4] P. Erdős and H. Heilbronn, On the addition of residue classes mod $p$, Acta Arith. 9 (1964), 149-159.

[5] W. Gao, On the size of additive bases of finite groups, preprint, October 1997.

[6] Y. O. Hamidoune, Adding distinct congruence classes, Combin. Probab. Comput. 7 (1998), 81-87.

[7] Y. O. Hamidoune and G. Zémor, On zero-free subset sums, Acta Arith. 78 (1996), $143-152$.

[8] Y. O. Hamidoune, A. S. Lladó and O. Serra, On sets with a small subset sum, Combin. Probab. Comput., to appear. 
[9] E. Lipkin, Subset sums of sets of residues, in: Conference on the Structure Theory of Set Addition, CIRM, Marseille, 1993, 187-197.

[10] H. B. Mann, Addition Theorems, 2nd ed., R. E. Krieger, New York, 1976.

[11] H. B. Mann and J. E. Olson, Sums of sets of elements in the elementary abelian group of type $(p, p)$, J. Combin. Theory 2 (1967), 275-284.

[12] H. B. Mann and Y. F. Wou, Addition theorem for the elementary abelian group of type $(p, p)$, Monatsh. Math. 102 (1986), 273-308.

[13] J. E. Olson, An addition theorem modulo p, J. Combin. Theory 5 (1968), 45-52.

[14] —, Sums of sets of group elements, Acta Arith. 28 (1975), 147-156.

[15] C. Peng, An addition theorem in elementary abelian groups, J. Number Theory 27 (1987), 58-62.

Department of Computer Science and Technology

University of Petroleum

Shangping Shiuku Road

Beijing 10200, China

E-mail:wdgao@publlc.fhnet.cn.net
Université P. et M. Curie

E. Combinatoire

Case 189

4, Place Jussieu

75252 Paris Cedex, France

E-mail: yha@ccr.jussieu.fr

Received on 3.3.1998

and in revised form on 21.9.1998 